\title{
COnE).(OES
}

CIÊNCIA E TECNOLOGIA

\section{O QUE CONHECEMOS DE TODOS O COSMOS?}

\author{
Hermano ENDLich SCHNEIDER VElte ${ }^{1}$ \\ ${ }^{1}$ Universidade Federal de Ouro Preto - UFOP \\ $<$ hermano.velten@ufop.edu.br>
}

DOI: https://doi.org/10.21439/conexoes.v13i4.1864

\begin{abstract}
Resumo. As observações astronômicas realizadas durante o eclipse solar ocorrido em Sobral em 29 de maio de 1919 constataram que a Teoria da Relatividade Geral de Einstein corretamente predizia o fenômeno da deflexão da luz. Este grande feito coloca esta teoria em um patamar único na história da ciência, do qual nunca mais saiu. A observação de outros fenômenos astronômicos, também preditos pela teoria de Einstein, ao longo do século XX, nos fazem ter a certeza absoluta que esta é a teoria que descreve a interação gravitacional. No âmbito cosmológico, no entanto, ao aplicar as equações da relatividade geral para a dinâmica de um modelo de universo homegêneo, isotrópico e em expansão aos mais recentes dados observacionais verifica-se que apenas 5\% do universo é composto por partículas conhecidas. A natureza e origem dos restantes $95 \%$ do conteúdo do universo, denominado de setor escuro, representa um dos grandes problemas que a ciência enfrentará no século XXI. Nosso objetivo nesse artigo é discutir sobre alguns fatos que promoveram a transição da gravitação Newtoniana para a relatividade geral e mostrar como os conceitos de matéria escura e energia escura surgiram ao longo do século XX. Com isso, seremos levados a uma visão geral do conteúdo material do universo e sua evolução dinâmica.
\end{abstract}

Palavras-chaves: Teoria da Relatividade Geral, Cosmologia, Matéria escura, Energia escura.

\begin{abstract}
The astronomical observations performed at the occasion of the solar eclipse at Sobral on the May 29th 1919 confirmed Einstein's General Relativity (GR) predictions about the light deflection. This seminal achievement brought GR to a very special place in Science history remaining there until today. Along the XXth century the observation of other astronomical phenomena, also predicted by GR, gives us the certainty that GR is the correct theory describying the gravitational interaction. In cosmology, however, when one applies the GR equations for the dynamics of a homogeneous, isotropic and expanding universe to the most recent observational data one verifies that only $5 \%$ of the universe is composed by known particles. The nature ans origin of the remaining $95 \%$ of the universe's energy budget, the dark sector of the universe, represents one of the greatest scientific challenges for the XXIst century. Our goal in this article is to discuss some basic facts that led the transition from the Newtonian gravity to GR and to show how the concepts of dark matter and dark energy arose along the XXth century. Hence, we promote a discussion about the universe's matter content and its dynamical evolution.
\end{abstract}

Keywords: General Relativity, Cosmology, Dark Matter, Dark Energy.

\section{Gravitação Universal de Newton e seu pri- meiro problema}

Muito antes de investigar o que há escondido na imensidão do universo o homem é, na verdade, um grande admirador do céu. De fato, todas as antigas culturas possuíam algum tipo de representação própria para o que hoje chamamos de constelações. No entanto, a par- tir da prática sistemática do método científico, mesmo que de maneira incipiente com Ptolomeu, Tycho Brahe e Johannes Kepler, mas sobretudo, sendo o método científico em si propriamente empregado por Galileu Galilei, temos o alvorecer da astronomia moderna que fatalmente nos conduziria ao entendimento atual dos cosmos em termos físicos e matemáticos. Com Isaac New- 
ton surge uma teoria para a gravitação com predições de fenômenos astronômicos testáveis sob o ponto de vista observacional. Ao longo do século XIX, apesar da notória eficácia da teoria da gravitação Universal de Newton, eis que surgem duas observações, relacionadas a aspectos físicos muito peculiares das órbitas dos planetas no sistema solar, que iniciariam uma revolução na astronomia. Primeiro, a órbita do planeta Mercúrio possuia um deslocamento angular considerável a cada revolução. Este fenômeno é comumente chamado de avanço do periélio de Mercúrio e não era compatível com a teoria de Newton para a gravitação (VERRIER 1859). Apesar de o teorema de Bertrand (BERTRAND. 1873) assegurar que uma órbita em um potencial do tipo Newtoniano deveria ser fechada - e portanto tal avanço não deveria ocorrer em um sistema de dois corpos - a influência gravitacional dos demais planetas induz um certo avanço mesmo nos domínios da teoria clássica de Newton. Ainda, em paralelo a este resultado, outra inconsistência também foi encontrada na órbita de Urano. Diante destes fatos e dada a certeza de que a teoria de Newton estava certa, a solução encontrada foi de postular a existência de outros dois planetas, próximos a Mercúrio e Urano, que explicariam tais anomalias. Próximo a Urano foi encontrado o planeta Netuno. No entanto, nunca encontrou-se outro planeta próximo a Mercúrio. Ou seja, havia, a partir deste episódio, uma importante evidência que colocava sob suspeita a teoria da gravitação Universal de Newton. A predição teórica Newtoniana de um avanço do periélio da órbita de Mercúrio obtida por Urban Le Verrier de 526, 7 segundos de arco por século era insuficiente para alcançar o valor observado. Medidas realizadas principalmente por le Verrier indicavam uma precessão de 565 segundos de arco por século. Permanecia uma diferença de 38,3 segundos de arco por século que não poderia ser ignorada e que também não estava associada a erros de medida que colocava sob forte suspeita a validade da teoria Newtoniana (veja uma detalhada discussão em (MIRANDA, 2019)).

\section{A Teoria da Relatividade geral de Einstein e seus problemas}

Façamos agora um grande salto na cronologia dos fatos diretamente para o início do século XX. Albert Einstein propõe sua aclamada teoria da relatividade geral (TRG) com uma predição teórica que corretamente ajustava a questão do avanço do periélio da órbita de Mercúrio. Assim, um problema que atormentava a astronomia por décadas acabava de ser solucionado. Dentre outras predições da teoria de Einstein havia ainda o fenômeno da deflexão da luz devido à curvatura do espaço tempo.
Por exemplo, a luz de uma estrela, ao tangenciar um objeto como o Sol, sofreria um desvio de sua trajetória retilínea e alcançaria outra rota. Em 29 de Maio de 1919 uma equipe da Royal Astronomical Society obtém medidas da deflexão da luz durante o eclipse ocorrido na cidade de Sobral/CE. A TRG passa a ser celebrada. A TRG ainda oferecia a predição de novos efeitos gravitacionais iniciando uma nova era para a pesquisa teórica e observacional. Trata-se do capítulo inicial de uma revolução no conhecimento da interação gravitacional com consequências diretas até os dias atuais.

Até hoje não houve nenhum experimento que foi capaz de contradizer as predições da TRG. Além da precessão da órbita de Mercúrio e da predição do desvio da luz havia ainda um terceiro teste (chamado de teste clássico) da TRG. A hipótese levantada por Einstein, segundo sua teoria, é a de que fótons mudariam sua frequência desde sua emissão até sua captação por influência da interação gravitacional. Em 1959 foi realizado um experimento para testar a mudança da frequência de um fóton gama emitido por uma fontes radioativas do elemento Ferro 57 (contendo 26 prótons e 31 nêutrons) ao atravessar o campo gravitacional da Terra. Nesse caso, o campo gravitacional foi o correspondente a um prédio de 4 andares (cerca de 22,5 metros). Os pesquisadores Robert Pound e Glen Rebka confirmaram que, de fato, havia uma mudança na frequência destes fótons compatível com a predição da TRG (POUND; JR, 1959).

Vários outros testes foram realizados desde a segunda metade do século XX até os dias atuais. Poderíamos também citar o efeito Shapiro (um atraso no tempo de viagem de sinais no sistema solar), a observação da queda do período orbital do pulsar binário de Hulse-Taylor, a própria verificação direta da existência das ondas gravitacionais e a recente imagem do buraco negro no centro da galáxia M87 como grandes triunfos da TRG. Assim, pode-se dizer que a TRG é uma teoria confiável, robusta e capaz de descrever fenômenos gravitacionais de maneira satisfatória. Estaríamos então em uma situação de conforto, onde não há mais a necessidade de buscar um melhor entendimento da interação gravitacional?

\section{Observações astronômicas que levaram ao conceito de matéria escura}

Fritz Zwicky é um dos mais importantes nomes da astronomia da primeira metade do século XX. No ano de 1933 ele realizou observações das velocidades de galáxias pertencentes à um aglomerado de galáxias denominado COMA. Trata-se de um aglomerado rico, possui- 
dor de mais de mil galáxias identificadas e caracterizadas. Os resultados de Zwicky indicavam que a energia cinética de todo o aglomerado, calculada via a obtenção das velocidades destas galáxias, não era compatível com a quantidade de energia potencial gravitacional, obtida através da contagem de objetos e inferência de sua massa. As quantidades de energia cinética $(\mathrm{K})$ e potencial gravitacional (U) devem obedecer ao chamado teorema do virial $2 \mathrm{~K}+\mathrm{U}=0$. Zwicky deduziu que havia uma falta de matéria observada para explicar as altas velocidades encontradas. Em outras palavras, deveria haver naquele sistema muito mais matéria do que podia ser observada no espectro eletromagnético. Com esse resultado nascia o conceito de matéria escura. Tratase de algum tipo de matéria capaz de interagir gravitacionalmente com as demais partículas e campos, mas que não emite nenhum tipo de radiação eletromagnética, logo, não é detectável.

Um segundo episódio na astronomia do século XX ocorre quando a astrônoma Vera Rubin mede a velocidades de rotação do disco de galáxias e, de maneira similar à Zwicky, conclui que as altas velocidades das estrelas não poderiam ser explicadas pela teoria Newtoniana com base na quantidade de matéria diretamente observada. É importante frisar que a dinâmica de galáxias e aglomerados não necessita de uma abordagem relativística. O fator de Lorentz para estes sistemas é praticamente igual à unidade. Ainda, estes não são objetos astrofísicos compactos, como estrelas de nêutrons e buracos negros, onde os efeitos da curvatura do espaço-tempo são fundamentais para a descrição da estrutura hidrodinâmica destes objetos (via equações de Tolmann-Oppenheimer-Volkoff) o que, neste caso, faria o uso da TRG imprescindível.

Outro fenômeno astrofísico que também indica a existência de matéria escura é o de lenteamento gravitacional. Neste caso, trata-se de uma manifestação intrínseca da TRG onde a deflexão dos raios de luz vindos de um objeto astrofísico distante são lenteados, por exemplo, por uma galáxia. Através da medição da forma e magnitude com que a galáxia curva a trajetória desta luz é possível reconstruir o seu perfil de massa. Esta técnica também tem confirmado a hipótese da existência de matéria escura e, ainda, fornecido informação precisa sobre o perfil de distribuição da matéria escura em sistemas como galáxias e aglomerados de galáxias.

Fora do ambiente astrofísico a cosmologia fornece indicações fortes sobre a existência da matéria escura. O universo possui 13,8 bilhões de anos. Entendemos que o processo de formação de estruturas cósmicas ocorreu de maneira hierárquica, ou seja, as menores es- truturas, como proto-galáxias, se formaram em um primeiro momento e a posterior evolução guiada pela interação gravitacional deu origem às estruturas maiores como galáxias e aglomerados de galáxias. As observações da radiação Cósmica de Fundo nos revelam que a magnitude das flutuações na distribuição de matéria, quando o universo possuía cerca de 380.000 anos, era da ordem de 1 parte em 100.000. Trata-se de um pequeno desvio da homogeneidade, mas fundamental para explicar a origem do padrão não homogêneo observado atualmente. Assim, ao confiar que a TRG conduz a evolução das perturbações de matéria deste momento inicial até os dias atuais, encontra-se uma grande incompatibilidade. A TRG sozinha não seria capaz de explicar o surgimento de estruturas virializadas que destoam do padrão de homogeneidade na distribuição de matéria como estrelas e galáxias. Faltaria tempo para que esse processo ocorresse. Assim, assume-se que a matéria escura exista no universo e tenha papel crucial ao impulsionar o processo de formação de estruturas. Novamente, ao confiar na TRG, é fundamental conceber a existência da matéria escura.

\section{Observações astronômicas que levaram ao conceito de energia escura}

A maneira como entendemos o universo mudou radicalmente no fim dos anos 1990. Até então estava claro que o universo deveria ser composto por matéria escura, radiação (como fótons e neutrinos relativísticos) e matéria bariônica ${ }^{1}$. Um problema claro deste tipo de universo é que ele seria muito jovem. Dificilmente teria mais do que 10 bilhões de anos. Ao mesmo tempo, se os modelos astrofísicos estivessem corretos, já se estimava que a idade dos aglomerados globulares poderia ser de 13 bilhões de anos. Havia então uma clara contradição na possibilidade de que o universo fosse mais novo do que alguns objetos que ele contém.

O desenvolvimento das observações das explosões de Supernovas indicou algo inesperado. Estes eventos apresentavam magnitudes abaixo do esperado. A explicação desta observação fez uso da hipótese que o Universo passa por uma fase de expansão acelerada (RIESS et al., 1998). Contrariamente ao esperado devido à natureza atrativa da atração gravitacional, uma expansão acelerada deveria ser ocasionada por algum tipo de matéria com propriedades anti-gravitacionais. A este tipo de entidade deu-se o nome de energia escura.

\footnotetext{
${ }^{1} \mathrm{O}$ termo matéria bariônica é um jargão utilizado para representar toda a matéria conhecida, uma vez que os bárions (partículas pesadas como prótons e nêutrons) contribuem muito mais do que mésons (partículas de massa intermediária) e léptons (partículas leves).
} 
$\mathrm{Na}$ formulação da TRG para um universo em expansão, a energia escura pode ser compreendida com um fluido com pressão (suficientemente) negativa. Basta lembrar que na TRG a pressão também "gravita", ou seja, ela é capaz de induzir uma modificação no espaçotempo e, consequentemente, determinar sua dinâmica. De maneira equivalente ao uso de um fluido com pressão negativa, a famosa constante cosmológica de Einstein (um objeto geométrico introduzido por Einstein na TRG para evitar a contração e colapso do universo) também seria capaz de explicar tal fenômeno, mas existe uma séria inconsistência entre seu valor teórico e o que seria necessário do ponto de vista observacional.

\section{O inventário cósmico}

Como resumo do que foi exposto até aqui chegamos à conclusão que matéria e energia escuras devem compor o inventário cósmico. Mas qual seria a proporção destas componentes? Antes de responder a esta pergunta é importante frisar que a dinâmica de um universo em expansão prevê que a densidade de suas componentes dependa do tempo. Assim, a composição do universo não permaneceu constante desde seu início. Uma aproximação usual em cosmologia utiliza a ideia de que a expansão cósmica, acima da escala de homogeneidade $\sim 300$ Mpc ( $\left.1 \mathrm{Mpc} \equiv 3,0857 \times 10^{19} \mathrm{~km}\right)$, pode ser idealizada através da fluido-dinâmica. A dependência temporal da densidade de cada componente cósmica depende de sua equação de estado onde sua pressão efetiva é relacionada à sua densidade. A combinação de todas as fontes observacionais indica que a distribuição do inventário cósmico obedece a seguinte proporção: Todas as partículas relativísticas correspondem a $0,005 \%$; a matéria comum, dita bariônica, a quase 5\%; a matéria escura a aproximadamente $25 \%$ enquanto que a energia escura 70\% (COLLABORATION, 2018). Estes números refletem nossa ignorância sobre o Universo. A grande maioria $(95 \%)$ da atual composição cósmica está distribuída nas componentes escuras das quais não temos conhecimento sobre sua natureza e origem.

\section{A expansão do Universo e a Teoria da Rela- tividade Geral}

O trabalho de Edwin Hubble no fim da década de 1920 foi crucial para o desenvolvimento da cosmologia moderna. Primeiramente, suas observações foram importantes para certificar a existência de galáxias além da nossa Via Láctea. Assim, ao aumentar o número de galáxias observadas, ele estabeleceu um ingrediente chave para a cosmologia moderna que é a expansão do uni- verso. Hubble verificou que galáxias se distanciam das outras com uma velocidade proporcional à sua distância. Para descrever esse processo é preciso parametrizar a expansão do Universo em termos do fator de escala cósmico $a(t)$. Dessa maneira, a distância física $\mathbf{r}_{\mathbf{f}}$ entre dois objetos em um certo instante $t$ pode ser escrita como $\mathbf{r}_{\mathbf{f}}=a(t) \mathbf{r}_{\mathbf{0}}$, onde $\mathbf{r}_{\mathbf{0}}$ é uma distância fixada em um instante $t_{0}$ que pode ser identificado com o tempo presente. Na nossa notação, o sub-índice 0 denota o valor de uma quantidade física medida hoje. Calculando a derivada temporal $\dot{\mathbf{r}}_{\mathbf{f}}$ obtém-se ${ }^{2}$

$$
\dot{\mathbf{r}}_{\mathbf{f}} \equiv \mathbf{v}_{\mathbf{f}}=H(t) \mathbf{r}_{\mathbf{f}}
$$

onde $H(t)$ é a taxa de expansão de Hubble

$$
H(t)=\frac{\dot{a}(t)}{a(t)} .
$$

Se esta relação é tomada no tempo presente com $H\left(t_{0}\right) \equiv H_{0}$, a relação (1) torna-se a famosa lei de Hubble, sendo $H_{0}$ a chamada constante de Hubble. Recentemente, esta nomenclatura foi modificada para incluir o nome de Lemaître pois ela já havia surgido em trabalhos anteriores à Hubble (LEMAîTRE, 1927).

É útil normalizar o valor do fator de escala hoje como a unidade $a_{0}=1$. Com esta convenção o fator de escala é relacionado com o parâmetro do desvio para o vermelho (redshift) cósmico $z$ por meio da definição $a(t)=(1+z)^{-1}$. Assim, o tempo presente corresponde à $z=0$. $\mathrm{O}$ passado longínquo ocorre no limite $z \rightarrow \infty(a \rightarrow 0)$. O limite futuro $a \rightarrow \infty$ ocorreria em $z=-1$.

A TRG é a teoria padrão para descrever os processos que envolvem a interação gravitacional. Nossa ideia daqui em diante é fazer uma breve introdução de seu aparato ferramental. Existem ótimos livros sobre a TRG mas, em sua grande maioria, são escritos na língua inglesa. Uma ótima referência, em língua portuguesa, sobre a TRG pode ser encontrada em (HENRIQUES. 2009).

O ponto de partida será introduzir a chamada equação de Einstein

$$
R_{\mu \nu}-\frac{1}{2} g_{\mu \nu} R+\Lambda g_{\mu \nu}=8 \pi G T_{\mu \nu}
$$

Não é o objetivo deste trabalho deduzir a equação acima. O elemento chave de uma geometria é o tensor métrico $g_{\mu \nu}$. A partir dele podemos calcular o tensor de Ricci $R_{\mu \nu}$ e o escalar de Ricci $R$. O fator $\Lambda$ é a constante cosmológica. Os índices gregos percorrem

\footnotetext{
${ }^{2}$ Temos em mente o chamado tempo cósmico $t$.
} 
os valores $\mu, \nu \rightarrow 0,1,2,3$. Assim, um objeto do tipo $R_{\mu \nu}$ deve ser idealizado como uma matriz $4 \mathrm{x} 4$. Todo o lado esquerdo de (3) representa a geometria do espaçotempo. A parte do conteúdo material é representada pelo tensor energia-momento $T_{\mu \nu}$ que se encontra ao lado direito desta equação. $G$ é a mesma constante da gravitação de Newton. A equação de Einstein descreve, de uma maneira bem geral, a interação entre a distribuição de matéria com a geometria do espaço-tempo. E como encontrar soluções desta equação? O primeiro passo é impor a simetria adaptada ao problema físicos que se tem em mãos. Na cosmologia padrão esta simetria é motivada pelo Princípio Cosmológico, que diz que o universo é homogêneo e isotrópico em grandes escalas. Assim, ao exigir este tipo de simetria passa-se a fixar a métrica do espaço-tempo como a de FriedmannLemaitre-Robertson-Walker (FLRW)

$$
d s^{2}=-d t^{2}+a(t)^{2}\left[\frac{d r^{2}}{1-k r^{2}}+d \Omega^{2}\right],
$$

onde a constante $k$ representa a curvatura espacial. Seguindo a notação usual, utilizamos unidades onde a velocidade da luz é a unidade $c=1$. O objetivo final é, a partir da eq. de Einstein, determinar a forma funcional do fator de escala $a(t)$. Utilizando (4) na eq. (3) obtém-se as chamadas equações de Friedmann

$$
H^{2}-\frac{k}{a^{2}}=\frac{8 \pi G}{3} \sum_{i} \rho_{i}+\frac{\Lambda}{3},
$$

sendo $\rho_{i}$ a densidade de energia associada a uma componente $i$. Assume-se que cada componente conservase, ou seja, não interage com as demais via troca de energia ou momento. Dessa forma, elas obedecem individualmente a equação da continuidade

$$
\dot{\rho}_{i}+3 H\left(\rho_{i}+p_{i}\right)=0 .
$$

Esta equação determina a evolução temporal da densidade do fluido $i$ uma vez que sua equação de estado $p_{i}\left(\rho_{i}\right)$ é conhecida.

Para cada componente define-se um parâmetro de densidade fracionária

$$
\Omega_{i}(z)=\frac{\rho_{i}(z)}{\rho_{c 0}}, \quad \text { with } \quad \rho_{c 0}=\frac{3}{8 \pi G} H_{0}^{2},
$$

onde $\rho_{c 0}$ é a densidade crítica do universo hoje. A partir desta definição também encontra-se o valor da densidade fracionária da componente $i$ hoje, ou seja, $\Omega_{i 0}=\rho_{i}(0) / \rho_{c 0}$. De uma maneira similar vamos definir as quantidades análogas para as demais componentes

$$
\Omega_{k 0}=-\frac{k}{H_{0}^{2}} \quad \text { e } \quad \Omega_{\Lambda 0}=\frac{\Lambda}{3 H_{0}^{2}}
$$

Ao assumir que a composição do universo consiste de radiação $(i=r)$ e matéria $(i=m)$, a Eq. (5) é reescrita como

$$
\begin{array}{r}
H^{2}(z)=H_{0}^{2}\left[\Omega_{r 0}(1+z)^{4}+\Omega_{m 0}(1+z)^{3}\right. \\
+\Omega_{k 0}(1+z)^{2}+\Omega_{\Lambda 0} .
\end{array}
$$

Com esta expressão um modelo de referência pode ser construído. A tarefa, a partir da Eq. (6), é obter a densidades fracionárias de radiação $\Omega_{r 0}$, de matéria $\Omega_{m 0}$ (que é composta por uma parte de matéria bariônica $\Omega_{b 0}$ e matéria escura $\Omega_{d m 0}$ ), da curvatura espacial $\Omega_{k 0}$ e da energia escura $\Omega_{\Lambda 0}$. Ainda, é fundamental conhecer a constante de Hubble $H_{0}$. A obtenção de dados astronômicos precisos é fundamental para a determinação destes parâmetros cosmológicos. A seguir, vamos discutir quais foram (e tem sido) alguns dos principais resultados observacionais que ajudaram a pavimentar o caminho até a formulação de um modelo cosmológico de concordância.

As observações da Radiação Cósmica de Fundo (RCF) foram fundamentais para estabelecer alguns fatos. Dentre estes podemos citar a temperatura de corpo negro associada à esta distribuição de radiação. Assim, sabemos com grande precisão que a densidade fracionária da radiação hoje é de $\Omega_{r 0}=9,29 \times 10^{-5}$. A RCF também é fundamental para determinar que o universo não admite um valor considerável de curvatura. Em outras palavras, temos, na prática, que $\Omega_{k 0} \approx 0$. Assumindo um universo plano $\Omega_{k 0}=0$ e fazendo $z=0$ em (6) a quantidade de energia escura pode ser escrita como $\Omega_{\Lambda 0}=1-\Omega_{m 0}$. Estes resultados simplificam o número de parâmetros livres da Eq. (6) para apenas dois, $H_{0}$ e $\Omega_{m 0}$. Por fim, o conjunto de diversas observações leva a um cenário onde $\Omega_{m 0} \approx 0.3$. É importante lembrar, no entanto, que a quantidade de bárions está incluída em $\Omega_{m 0}$. Dentro do cenário da bariogênese cósmica determina-se $\Omega_{b 0} \approx 0.05$, o que significa dizer que cerca de $25 \%$ do cosmos atual é composto por matéria escura. Com isso, chegamos ao resultado $\Omega_{\Lambda 0} \approx 0.7$.

$\mathrm{O}$ valor exato do parâmetro de Hubble $H_{0}$ é tema de grande debate atual na literatura. Existem distintas formas de se obter $H_{0}$. A partir das análises estatísticas com dados cosmológicos, justamente o cenário descrito no parágrafo anterior, encontra-se um valor próximo a $H_{0}^{\text {cosmo }} \approx 69 \mathrm{~km} / \mathrm{s} / \mathrm{Mpc}$ (COLLABORATION, 2018). Por outro lado, a velocidade de recessão de galáxias e outros objetos astrofísicos próximos à nossa galáxia pode ser determinada diretamente. Este tipo de análise 
indica valores $H_{0}^{\text {astro }} \approx 73 \mathrm{~km} / \mathrm{s} / \mathrm{Mpc}$ (SHANKS; HOGARTH; METCALFE, 2018). Trata-se de uma tensão considerável mesmo quando as barras de erros associadas à estes valores são levadas em consideração.

Ao utilizar estes números para os parâmetros cosmológicos podemos visualizar como as densidades de energia evoluíram desde o universo primordial até o hoje $z=0$. Assim como demonstrado na Fig.1, o universo era dominado pela radiação - o termo $\Omega_{r 0}(1+z)^{4}$ domina o lado direito de (6) - em um momento anterior ao desvio para o vermelho $z \approx 3300$. Isso corresponde ao momento que o universo tinha apenas 60.000 anos de existência. É bom lembrar que a idade do universo não segue uma relação linear com o desvio para o vermelho. Deste momento até o desvio para o vermelho $z \approx 0.3$, correspondendo a cerca de 3,5 bilhões de anos atrás, a dinâmica cósmica é dominada pela matéria. Isso é fundamental para a execução do processo de formação de estruturas pois nessa etapa que galáxias e aglomerados se formam. A Fig.2 é equivalente à Fig.1, porém mostra mais detalhes sobre o período recente onde a energia escura passa a dominar a dinâmica da expansão cósmica. A densidade fracionária da energia escura, na forma de uma constante cosmológica, é representada pela linha sólida horizontal. A partir deste instante a expansão acelerada do universo impede a aglomeração de matéria nas escalas cósmicas. Isso faz com os aglomerados de galáxias sejam as maiores estruturas virializadas observadas no universo.

\section{Comentário Final}

O intuito deste trabalho é fazer um breve panorama sobre a aplicação da Teoria da Relatividade Geral no estudo da cosmologia e as consequências deste formalismo que são, a saber, a aceitação da existência de duas componentes misteriosas denominadas Matéria Escura e Energia Escura. Estes resultados não enfraquecem a ideia de que a TRG representa a maneira de descrever a interação gravitacional. No entanto, estes fatos revelam que ainda há um longo caminho a se percorrer até conhecermos o que há em todo cosmos. A investigação dos mistérios do universo está nos seus primórdios e muitos outros novos resultados ainda estão por vir no século XXI.

\section{REFERÊNCIAS}

BERTRAND, J. Théoreme relatif au mouvement d'un point attiré vers un centre fixe. CR Acad. Sci, v. 77, n. 849-853, p. 2, 1873.

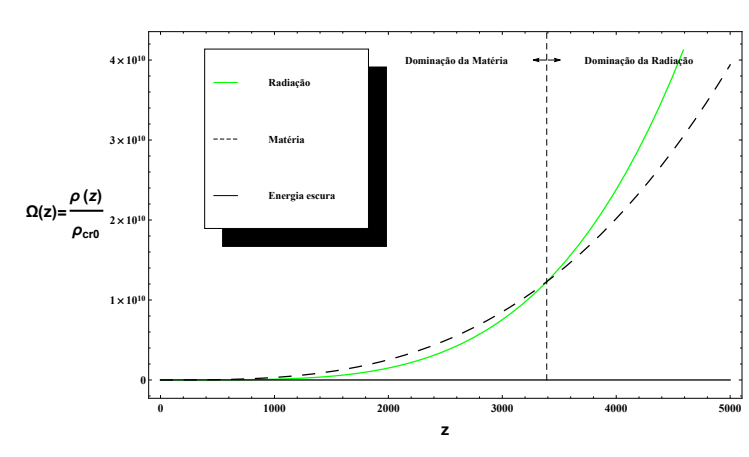

Figura 1: Evolução da densidade fracionária de energia da radiação (verde), matéria (tracejado) e energia escura (linha preta horizontal) como uma função do desvio para o vermelho (z).

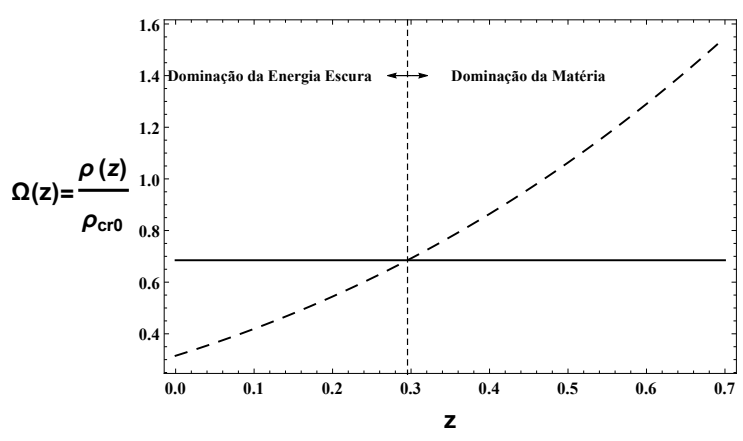

Figura 2: Evolução da densidade fracionária de matéria (tracejado) e energia escura (linha preta horizontal) como uma função do desvio para o vermelho $(\mathrm{z})$

COLLABORATION, P. Planck 2018 results. vi. cosmological parameters. [e-Print: arXiv:1807.06209]. 2018.

HENRIQUES, A. B. Teoria da Relatividade Geral: Uma Introdução. 1. ed. São Paulo: Instituto Superior Técnico, 2009.

LEMAîTRE, G. Annales de la société scientifique de bruxelles. A47, 49 (1927) [Translated into English inMNRAS, v.41, p.483 1931]. 1927.

MIRANDA, O. D. Avanço do periélio de mercúrio-o primeiro sucesso da teoria da relatividade geral de einstein. Conexões-Ciência e Tecnologia, v. 13, n. 2, p. 7-20, 2019.

POUND, R. V.; JR, G. A. R. Gravitational red-shift in nuclear resonance. Physical Review Letters, APS, v. 3, n. 9, p. 439, 1959. 
RIESS, A. G.; FILIPPENKO, A. V.; CHALLIS, P.; CLOCCHIATTI, A.; DIERCKS, A.; GARNAVICH, P. M.; GILLILAND, R. L.; HOGAN, C. J.; JHA, S.; KIRSHNER, R. P. et al. Observational evidence from supernovae for an accelerating universe and a cosmological constant. The Astronomical Journal, IOP Publishing, v. 116, n. 3, p. 1009, 1998.

SHANKS, T.; HOGARTH, L.; METCALFE, N. Gaia cepheid parallaxes and 'local hole'relieve h 0 tension. Monthly Notices of the Royal Astronomical Society: Letters, Oxford University Press, v. 484, n. 1, p. L64-L68, 2018.

VERRIER, U. L. Lettre de $\mathrm{m}$, le verrier à m: Faye sur la théorie de mercure et sur le mouvement du périhélie de cette planète. Comptes rendus hebdomadaires des séances de l'Académie des sciences, v. 49, n. 1, p. 379-383, 1859. 